\title{
Impurity binding energy for $\delta$-doped quantum well structures
}

\author{
V TULUPENKO ${ }^{\mathrm{a}}$, C A DUQUE ${ }^{\mathrm{b}}, \mathrm{R}^{\mathrm{D}}$ DEMEDIUK ${ }^{\mathrm{a}}$, O FOMINA $^{\mathrm{a}}, \mathrm{V}$ AKIMOV $^{\mathrm{a}, \mathrm{b}, *}$, \\ $V_{\text {BELYKH }}{ }^{\mathrm{a}}$, T DMITRICHENKO ${ }^{\mathrm{a}}$ and V POROSHIN ${ }^{\mathrm{c}}$ \\ ${ }^{a}$ Department of Physics, Donbass State Engineering Academy, Shkadinova 72, 84313 Kramatorsk, Ukraine \\ ${ }^{\mathrm{b}}$ Grupo de Materia Condensada-UdeA, Instituto de Fisica, Facultad de Ciencias Exactas y Naturales, \\ Universidad de Antioquia UdeA; Calle 70 No. 52-21, Medellin, Colombia \\ ${ }^{\mathrm{c}}$ Institute of Physics of National Academy of Science, $80028 \mathrm{Kiev}$, Ukraine
}

MS received 26 October 2013

\begin{abstract}
The binding energy of an impurity delta layer situated either in the centre or at the edge of a quantum well $(\mathrm{QW})$ is theoretically considered for the example of $n$-type $\mathrm{Si}_{0.8} \mathrm{Ge}_{0.2} / \mathrm{Si} / \mathrm{Si}_{0.8} \mathrm{Ge}_{0.2} \mathrm{QW}$ doped with phosphorus. Calculations are made for the case of not so big impurity concentrations, when impurity bands are not yet formed and it is still possible to treat impurity as isolated ones. It is shown on the base of self-consistent solution of Schrödinger, Poisson and electro-neutrality equations that impurity binding energy is dependent on the degree of impurity ionization and the most noticeably for the case of edge-doped QWs.
\end{abstract}

Keywords. Quantum well; impurity binding energy; delta-doping.

\section{Introduction}

Impurities in quantum wells (QWs) are extremely interesting object for investigation. For definiteness, we further deal with hydrogen-like impurities in rectangular QWs. We also consider neither the high impurity concentrations when there are tails of density of states in the forbidden gap, nor intermediate impurity concentrations, when impurity bands are emerged. All the attention of the paper will be paid to the case of not so big impurity concentration, when impurities can be treated as isolated ones. In this case, their binding energy is very sensitive to the position of the impurity in the QW, and to the width of the QW as it was first pointed out by Bastard (1981). Since then, a lot of other heterostructure parameters and external actions influencing the impurity binding energy (IBE) were studied in a big number of publications (mostly theoretically). Some of these references one can find in our work (Tulupenko et al 2011). But, among a big variety of factors influencing IBE there is one, in our knowledge, not yet studied - namely the distribution of carriers within QW due to ionization of impurity delta layer positioned inside the well. Owing to impurities are shallow, their ionization can be easily achieved either by relatively small external electric field or by increasing temperature. Once the delta layer is (partly) ionized, the new potential (hereafter we will call it a Hartree potential), created both by free electrons in space-quantized energy sub-bands and by ionized delta-layer, is superimposed on

\footnotetext{
*Author for correspondence (it-ogo@yandex.ru)
}

the original, not disturbed (with neutral impurity delta layer) rectangular QW energy profile. It means that a new QW with a new set of space-quantized energy levels and accordingly with a new impurity binding energy is created. Therefore, the aim of the paper is to present our results on investigation of this phenomenon. The paper is organized as follows. In §2, we ground our choice of the object of the study as well as ground and explain the parameters of the chosen structure. In $\S 3$, we briefly touch the method of calculation, $\S 4$ is devoted to discussion of the obtained results and $\S 5$ with concluding remarks.

\section{Comments on the chosen object}

As the object for our study, we used the $\mathrm{Si}_{0.8} \mathrm{Ge}_{0.2} / \mathrm{Si} /$ $\mathrm{Si}_{0.8} \mathrm{Ge}_{0.2}$ QWs doped with phosphorus and grown in 100 crystallographic direction. Our choice was conditioned by the following. First, it is n-type QW which is easier for calculations than $p$-type structure. Second, the similar structure was carefully investigated by Blom et al (2003). It means that we can compare our results with those obtained in that work when it is possible and we therefore, have additional possibility for checking our own results and approach. Third, it is known from Blom et al (2003) that phosphorus binding energy in such a structure (without chemical shift) is about $30 \mathrm{meV}$ - the range is easily reached experimentally contrary to, for instance, the GaAs/AlGaA-doped QWs, where dopant binding energy is in the range of a few $\mathrm{meV}$, that is not so convenient for experimental investigations. Another important circumstance is that to have the noticeable affect of the 
ionized delta layer on the IBE, one has to use the impurity concentration as high as possible (at the same time remembering that impurities must be treated as isolated ones). In other words, chosen concentration must be less than critical one $N_{\mathrm{M}}$ for the Mott metal-insulator transition, which can serve as a crude criterion between low and high concentrations and can be estimated from relation $a_{\mathrm{B}} N_{\mathrm{M}}^{1 / 2} \cong 0.37$ (Schmalz et al 1998). It is seen from here that to have the biggest concentration one has to use the material with smaller Bohr radii $a_{B}$. In our case, $\alpha_{\mathrm{B}} \approx 2 \mathrm{~nm}$ (Tulupenko et al 2013), whereas in the case of doped GaAs/AlGaA QW, it is more than 5 times bigger. Thus, the impurity concentration we use is $1.2 \times 10^{12} \mathrm{~cm}^{-2}$, which is about 3 times less than $N_{\mathrm{M}}$. If we assume that all the donors are ionized (that is not true even at $T=300 \mathrm{~K}$ ), then average distance between them is $d^{+}=N^{-1 / 2}>2 \times 1 \cdot 3 a_{\mathrm{B}}$. The inequality is justified the single impurity approach (Brum et al 1984) and it is fulfilled at both 77 and $300 \mathrm{~K}$.

In our calculations, we do not account for the background impurities in the barriers. It means that the number of free electrons in space quantized sub-bands is equal to the number of ionized impurities of the delta layer.

We calculated binding energies for centre- and edgedoped QWs with different widths: 5, 10 and $20 \mathrm{~nm}$ and with different thicknesses, $\delta$ of delta layer: $\delta=1,2$ and $3 \mathrm{~nm}$. We supposed the uniform (not Gauss) distribution of impurities along the growth direction (z-axis) of the structure within $\delta$.

\section{Method remarks}

To find impurity binding energy, one has to solve appropriate Schrödinger equation. But, before we describe our way of solution to the equation, we discuss some simplifying assumptions and make some remarks about parameters of the structure used.

\subsection{On the exchange and correlation interaction}

Strictly speaking, to omit the term responsible for exchange and correlation interaction in the Hamiltonian, the condition that distance between free electrons, $d_{\mathrm{e}}$ must exceed de Broglie wavelength, $\lambda$ has to be fulfilled: $d_{\mathrm{e}}>\lambda$. Here $\lambda=h /\left(m_{\|} k T\right)^{1 / 2}$ with $h$ and $k$ being the Planck and Boltzmann constants, respectively. For impurity concentration used and degree of ionization at 77 and $300 \mathrm{~K}$, this condition is not satisfied as it follows from our calculations (Tulupenko et al 2013) and we must, in principle, include the term responsible for the many electron phenomena in the Hamiltonian. We, however, did not do that because it was shown in Cartoixa et al (2005) and Rodriguez-Vargas et al (2006) (see also Tulupenko et al 2013) that taking into account exchange and correlation interaction for our concentration led to lowering Fermi level by a value as small as less than $1 \mathrm{meV}$. Therefore, we ignore exchange and correlation interaction in our calculations.

\subsection{On the random impurity distribution}

Usually at high impurity concentrations, Hartree potential is considered to be only $z$-dependent with $z$ coincided with the growth direction of the structure. For our relatively small impurity concentration, this requires additional grounding as inhomogeneous in the impurity location involves fluctuation in Hartree potential. It was shown by Shklovskii et al (1984) in their book that for the random impurity distribution, there exist the regions for which the fluctuation potential cannot be screened. Thus, one can conclude that if the maximum size of the regions, $r_{\mathrm{s}}$ is less than de Broglie wavelength, $\lambda$ of a free electron $r_{\mathrm{s}}<\lambda$, then the fluctuation can be ignored (see also Scolfaro et al 1994). In our case, the inequality is fulfilled both at 77 and $300 \mathrm{~K}$ as it was shown in the work (Tulupenko et al 2013). It is also worth mentioning that as far as we neglect background doping, the $r_{\mathrm{s}}$ is equal to the distance between ionized impurities, $r_{\mathrm{s}}=d^{+}$ (Shklovskii et al 1984). For those confined on the impurity electron, the real distribution of impurities is unimportant, if the spread of the impurity wave function is larger than the width of the delta layer, i.e. $1 \cdot 3 a_{\mathrm{B}}>\delta$. This condition is fulfilled for delta layer thicknesses 1 and $2 \mathrm{~nm}$ and it is not satisfied for $\delta=3 \mathrm{~nm}$. But to see the trend, we also made calculations for this width of the delta layer as well.

\subsection{About parameters of the structure used}

Following the work (Blom et al 2003), we neglect small differences in parallel electron effective masses and dielectric constants between barrier and QW materials and also do not take into attention their temperature changes. We suppose that sub-band energies are isotropic and parabolic in a QW plane and impurities are uniformly distributed within the delta layer.

\subsection{About valley and impurity level splitting}

Due to the lattice constants mismatch between well (Si) and barrier materials $\left(\mathrm{Si}_{0.8} \mathrm{Ge}_{0 \cdot 2}\right)$, the $\mathrm{Si} \mathrm{QW}$ is stressed in the QW plane that is equivalent to the uniaxial pressure applied in the growth direction of the structure. The strain is governed by the per cent content, $x$ of Ge and is indirectly taken into account in the depth of the $\mathrm{QW}=200 \mathrm{meV}$ in our case. Besides, the strain results in lifting valley degeneration of $\mathrm{Si}$ - two valleys go down and for others, it goes up in the energy scale (Bir et al 1974). For our Ge content, the gap between these valleys 
is more than $100 \mathrm{meV}$ (Van de Walle et al 1986) (depends on the value of chosen deformation potential). It means that transport effective mass in our calculations: $m *=2\left(m_{\|}^{2} m_{\perp}\right)^{1 / 3}$ with 2 being a number of lowest valleys. Here, $m_{\|}=0 \cdot 19 m_{0}$ (mass in plane of QW), $m_{\perp}=0.92 m_{0}$ (mass perpendicular to the plane of QW) and $m_{0}$ is a free electron mass. The valley splitting is also accompanied with splitting of impurity levels (Bir et al 1974). We however, do not take the splitting into account in our calculations of impurity binding energy. Instead, following the work (Blom et al 2003), we consider joint influence of splitting and chemical shift on impurity ionization energy later, while discussing the obtained results.

After grounding the above assumptions, the Hamiltonian is significantly simplified and we find the IBE in the effective mass approximation from self-consistent solution of Schrödinger, Poisson and electroneutrality equations. The main difficulty in solving the Schrödinger equation is unseparated variables in the Coulomb term. We used the approach developed by Vinter (1982). The idea is to expand unknown envelope function in terms of known (without Coulomb term) functions and in such a way to reduce differential equation to the set of algebraic ones. Some details of the procedure can also be found in Stopa et al (1989) and in our works (Tulupenko et al 2011, 2013). Here, we only note that all the calculations were executed iteratively. At the first step, the energy profile of QW was taken as a rectangular one with Hartree potential equal to zero (which corresponds to situation at $T=4 \mathrm{~K}$ ). The IBE found at this step was then used for calculations of Fermi level (from electro-neutrality equation) and Hartree potential (from Poisson equation). Then, solution of Schrödinger equation with Hamiltonian including the Hartree potential, gave the new value of IBE. This value was again used for calculations of new values of Fermi level and Hartree potential and so on. At all stages of calculations, the convergence of impurity binding energy, $E_{\mathrm{d}}$ was checked as a difference $\Delta$ between last and last but one values of $E_{\mathrm{d}}$. After the fourth to sixth stage, we came to $\Delta<0 \cdot 1 \mathrm{meV}$ and calculations were stopped.

\section{Results and discussion}

Results of calculations for temperatures 4, 77 and $300 \mathrm{~K}$ and for three QW widths (5, 10 and $20 \mathrm{~nm}$ ) are shown in table 1 for delta-layer thickness $1 \mathrm{~nm}$. At the beginning, we have to note that results for all QW widths of the centre-doped QWs at $T=4 \mathrm{~K}$ coincide fairly well with the data shown in figure 3 of the work (Blom et al 2003). For edge-doped $\mathrm{Si}_{0.8} \mathrm{Ge}_{0.2} / \mathrm{Si} / \mathrm{Si}_{0.8} \mathrm{Ge}_{0.2}$ QWs, we did not find in the literature, the results at $T=4 \mathrm{~K}$ similar to those in Blom et al (2003), but our data correspond qualitatively to Bastard's (1981) calculations for GaAs/AlGaAs materials. It all means that our approach is correct and we therefore, can start discussing new results - namely, the results at elevated temperatures. It is seen from table 1 that influence of the temperature (or, in other words, of Hartree potential) on IBE becomes more pronounced for wider QWs independently on place of doping. It is natural as bigger QW widths means lower initial IBEs (at $T=4 \mathrm{~K}$ ) (Bastard 1981; Blom et al 2003). As a result, Hartree's potential increment with temperature is obviously bigger for lower initial binding energies. But the most interesting in our understanding, are the results for edge-doped QWs. Indeed, as we have already mentioned, it was shown in many works (see some references in work (Tulupenko et al 2011)) that IBE for edge-doped QWs is considerably smaller than for centre-doped ones. And our results demonstrate that it is true only for low temperatures when all the impurities are neutral. Ionization of impurities is followed with increasing IBEs, and difference between them for centre- and edge-doped QWs becomes smaller. Let us have a closer look at these results. Figure 1, in which the energy profile of the QW with $20 \mathrm{~nm}$ width, first space-quantized levels and their wave functions with ground impurity state are shown, helps us to do that. We assume that it is the first space-quantized level, which mainly contributes to the formation of the impurity states, and the narrower is the QW, the bigger is the contribution. At low temperature $T=4 \mathrm{~K}$ (figure 1a), practically all the impurities are neutral. It means that there are no free electrons even in the first quantization sub-band and accordingly there is no charge redistribution in the whole QW. Therefore, the energy profile of QW is remained unchanged and interrelation between values of impurity binding energies for centre- and edge-doping corresponds to that obtained in the earlier works cited in Tulupenko et al (2011). The temperature increase is accompanied with the impurity ionization and with the emergence of

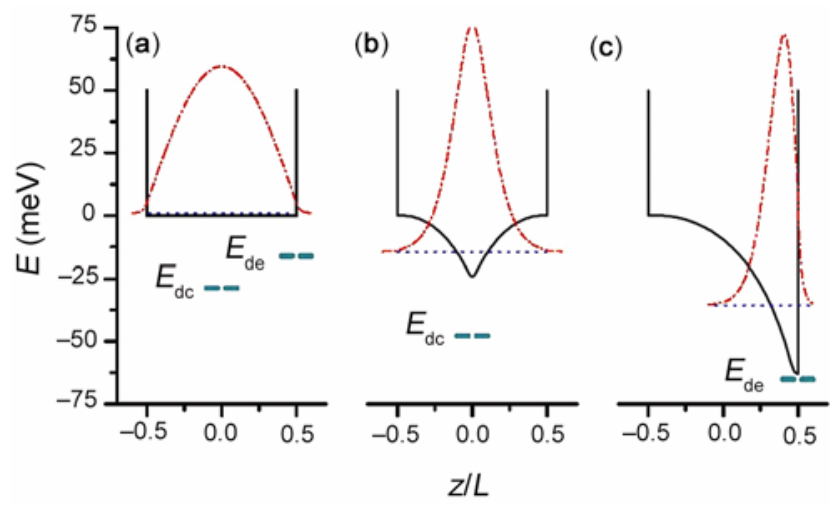

Figure 1. Energy profile - solid lines, 1 space quantization levels and their wave functions - dotted and dot-dashed lines, respectively, ground impurity states for centre doping. $E_{\mathrm{dc}}$ and edge doping $E_{\mathrm{de}}$ - dashed lines for $\mathrm{Si}_{0.8} \mathrm{Ge}_{0.2} / \mathrm{Si} \mathrm{QW}, L=20 \mathrm{~nm}$. (a) $T=4 \mathrm{~K}$; (b) $T=300 \mathrm{~K}$, centre-doped $\mathrm{QW}$ and (c) $T=$ $300 \mathrm{~K}$, edge-doped QW. Zero of energy is at the bottom of the $\mathrm{QW}$ at $4 \mathrm{~K}$. All wave functions are in the same scale. 
electrons in the space quantization sub-bands. Then, the potentials of ionized impurities and electrons in subbands give rise to the Hartree potential. It leads to the distortion of original (at $T=4 \mathrm{~K}$ ) QW energy profile. The picture looks like ionized delta layer digs out its own QW, which is superimposed on the original rectangular QW - figure 1(b) for centre doping and figure 1(c) for edge-doping. These new QWs have their own impurity ionization energies. Let us first consider and compare the centre-doping cases for different temperatures - figure 1(a) for $T=4 \mathrm{~K}$ and figure 1 (b) for $T=300 \mathrm{~K}$. One can see that the first quantized level emerges in the 'dug out' narrow appendix (figure 1b) and its wave function is noticeably changed with the temperature - it becomes bigger and narrower at the well centre. It results in larger localization of a free electron near the well centre with increasing temperature and consequently, in bigger IBE. It is worth noting that for QW widths of 5 and $10 \mathrm{~nm}$, the appendices are too shallow and they contain no spacequantized levels. It means that positions of first (and others) space-quantized levels practically are not changed with the temperature and it is the reason why IBEs for such narrow QWs are changed by so a small value. Now let us look at, and compare the results for the edge doping - figure 1(a) and (c). The temperature increases to $300 \mathrm{~K}$ involves modification of the energy profile of the QW by Hartree's potential. It results in the following. First, both the wave functions of the first energy quantization level on the whole and its maximum are shifted towards the QW edge, closer to the impurity atom. Second, the maximum of the wave function at $T=300 \mathrm{~K}$ is appreciably bigger than that for $T=4 \mathrm{~K}$. It all means that free electron is now nearer to the impurity atom that immediately results in the increase in the impurity binding energy. One can notice from table 1 that for edge-doped QWs with different widths, their IBEs are very close to each other at $T=300 \mathrm{~K}$. It can be explained as follows. The calculations show that in spite of the fact that for different QW widths the positions of first spacequantized levels in the energy scale are different at room temperature, their envelope functions are quite similar both in shape and in value. And it also justifies our attempt to explain the obtained results by considering the firsts space-quantized levels alone.

Now, let us discuss the obtained results for different thicknesses of a delta layer. On the base of general (symmetry) considerations, one can conclude that for centre-doped QWs, the width of the delta layer does not influence much on IBE because doping does not change the symmetry of the QW. Indeed, our results show that such conclusion is correct. Namely, an increase in thickness of delta layer from 1 to $3 \mathrm{~nm}$ results in a maximum decrease of IBE (for $L=20 \mathrm{~nm}$ ) less than $0.5 \mathrm{meV}$ at $T=300 \mathrm{~K}$. And, we therefore, do not discuss these results. More interesting are the results for edge-doped QWs, which are presented in table 2. Here the IBEs for edge-doped QWs with different thicknesses of delta layer and for different widths of QWs are shown at 4 and $300 \mathrm{~K}$. In our calculations, the coordinate $z$ of the middle of the delta layer for edge-doped QW was taken as $z=1-0 \cdot 5 \delta / L$. It follows from this, that it is not strictly correct to compare the results with the same thickness of the delta layer for different widths of QWs. The same is true even when comparing the results with different delta layer thicknesses for the same quantum well. Such comparisons can only be done for estimation purposes and to see the tendency. Thus, one can only compare the results at different temperatures with the same relative coordinate

Table 1. Impurity binding energies for centre-doped, $E_{\mathrm{dc}}(\mathrm{meV})$ and edge-doped, $E_{\mathrm{de}}(\mathrm{meV}) \mathrm{QWs}$ with different widths, $L(\mathrm{~nm})$ and at different temperatures, $T(\mathrm{~K})$.

\begin{tabular}{rccccccc}
\hline & \multicolumn{2}{c}{$E_{\mathrm{dc}}, \mathrm{meV}$ (centre-doped QWs) } & & \multicolumn{3}{c}{$E_{\mathrm{de}}, \mathrm{meV}$ (edge-doped QWs) } \\
\cline { 2 - 3 }$T(\mathrm{~K})$ & $L=5 \mathrm{~nm}$ & $L=10 \mathrm{~nm}$ & $L=20 \mathrm{~nm}$ & & $L=5 \mathrm{~nm}$ & $L=10 \mathrm{~nm}$ & $L=20 \mathrm{~nm}$ \\
\hline 4 & $37 \cdot 6$ & $32 \cdot 6$ & $29 \cdot 6$ & & $27 \cdot 5$ & $20 \cdot 4$ & $17 \cdot 0$ \\
77 & $37 \cdot 6$ & $32 \cdot 7$ & $30 \cdot 0$ & & $27 \cdot 9$ & $23 \cdot 3$ & $22 \cdot 0$ \\
300 & $37 \cdot 8$ & $34 \cdot 2$ & $33 \cdot 4$ & & $30 \cdot 5$ & $30 \cdot 2$ & $29 \cdot 6$ \\
\hline
\end{tabular}

Table 2. Impurity binding energies for edge-doped QWs with different thicknesses $(\delta=1,2$ and $3 \mathrm{~nm}$ ) of delta layer for different QW widths $(L=5,10$ and $20 \mathrm{~nm})$ and at temperatures, $T=4$ and $300 \mathrm{~K}, E_{\mathrm{de}}, \mathrm{meV}$.

\begin{tabular}{|c|c|c|c|c|c|c|}
\hline \multirow[b]{2}{*}{$\delta(\mathrm{nm})$} & \multicolumn{2}{|c|}{$E_{\mathrm{de}}, \mathrm{meV}$ for $L=5 \mathrm{~nm}$} & \multicolumn{2}{|c|}{$E_{\mathrm{de}}, \mathrm{meV}$ for $L=10 \mathrm{~nm}$} & \multicolumn{2}{|c|}{$E_{\mathrm{de}}, \mathrm{meV}$ for $L=20 \mathrm{~nm}$} \\
\hline & $T=4 \mathrm{~K}$ & $T=300 \mathrm{~K}$ & $T=4 \mathrm{~K}$ & $T=300 \mathrm{~K}$ & $T=4 \mathrm{~K}$ & $T=300 \mathrm{~K}$ \\
\hline 1 & $27 \cdot 5$ & $30 \cdot 5$ & $20 \cdot 4$ & $30 \cdot 2$ & $17 \cdot 0$ & $29 \cdot 6$ \\
\hline 2 & $32 \cdot 1$ & $34 \cdot 3$ & $24 \cdot 6$ & $33 \cdot 8$ & $18 \cdot 8$ & 33.9 \\
\hline 3 & $35 \cdot 6$ & $36 \cdot 7$ & $27 \cdot 6$ & $35 \cdot 0$ & $22 \cdot 2$ & $35 \cdot 4$ \\
\hline
\end{tabular}


of the delta layer. Nevertheless, we see from table 2 that the increase in the delta layer thickness is followed by increase in IBE. Such tendency can be explained by the fact that an increase in a delta layer thickness means a decrease in average distance between electrons in first space-quantized sub-band and impurity atom of the delta layer, which immediately results in increasing IBE.

As it was mentioned above, the chemical shift and splitting of the ground impurity state (due to the lattice mismatch) were not allowed for calculation of the impurity binding energy. To correct our results for these factors, we follow the logic and approach used in the work (Blom et al 2003) just for the same QW structure. It was shown that about $8 \mathrm{meV}$ are to be added to the impurity binding energy for the centre-doped QW and nothing added for the edge-doped QW (Blom et al 2003). These results were obtained for non-distorted QWs. As for our results for $T=300 \mathrm{~K}$, we suppose that for the centre-doped QWs, the same $8 \mathrm{meV}$ must be added. For the edge-doped QWs, one cannot directly state that these QWs are indeed edge-doped as it is seen from figure 1(c). They are rather doped somewhere between centre and edge of these new (dug out) QWs. Hence, this appears now that one must evaluate the value of the impurity offset to the QW width ratio and then using the data of figure 7 from the work (Blom et al 2003) to make a correction for chemical shift to the IBE. We estimate that correction to be about $7 \mathrm{meV}$.

\section{Conclusions}

In summary, we show that influence of ionization of impurity delta layer on IBE does really exist and depends on the place of the delta layer within a QW. Calculations were made for edge- and centre-doped QWs with different thicknesses of the delta layer and with different QW widths. The IBEs are enlarged with ionization of a delta-layer for all positions of the delta layer in a QW and the most noticeable increase is for edge-doped QWs. In this case, two factors contribute to this increase ionization of delta layer and accompanied it in central cell shift, whereas in the case of centre-doped QWs, only ionization of delta layer involves in the increase in IBE.

\section{Acknowledgements}

The work is partially supported by the UFBR grant $0113 U 000612$ and by Ukrainian Ministry of Education and Science. CAD is grateful to the Colombian Agencies COLCIENCIAS, CODI-Universidad de Antioquia (Estrategia de Sostenibilidad 2013-2014 de la Universidad de Antioquia) and Facultad de Ciencias Exactas y NaturalesUniversidad de Antioquia (CAD-exclusive dedication project 2012-2013). The work was developed with the help of CENAPAD-SP, Brazil.

\section{References}

Bastard G 1981 Phys. Rev. B24 4714

Bir G L et al 1974 Symmetry and strain-induced effects in semiconductors (New York: Wiley)

Blom A et al 2003 Phys. Rev. B68 165338

Brum J A et al 1984 Phys. Rev. B30 905

Cartoixa X et al 2005 Phys. Rev. B72 125330

Rodriguez-Vargas I et al 2006 J. Appl. Phys. 99033702

Schmalz K et al 1998 Phys. Rev. B57 6579

Scolfaro L M et al 1994 Phys. Rev. B50 8699

Shklovskii B I et al 1984 Electronic properties of doped semiconductors (Heidelberg: Springer)

Stopa M et al 1989 Phys. Rev. B40 8466

Tulupenko V et al 2011 J. Appl. Phys. 109064303

Tulupenko V et al 2013 Philos. Mag. Lett. 9342

Van de Walle C G et al 1986 Phys. Rev. B34 5621

Vinter B 1982 Phys. Rev. B26 6808 